\title{
Pretreatment Normal WM Magnetization Transfer Ratio Predicts Risk of Radiation Necrosis in Patients with Medulloblastoma
}

\author{
(D).H. Harreld, (D) P. Zou, (D) N.D. Sabin, (D) A. Edwards, (D). Han, (D). Li, (D). Bieri, (D) R.B. Khan, (D) A. Gajjar,
}

(D) G. Robinson, and (D)T.E. Merchant

o- $\equiv$

\begin{abstract}
BACKGROUND AND PURPOSE: Radiation necrosis, for which abnormal WM enhancement is a hallmark, is an uncommon complication of craniospinal irradiation in children with medulloblastoma. The magnetization transfer ratio measures macromolecular content, dominated by myelin in the WM. We investigated whether the pretreatment supratentorial (nonsurgical) WM magnetization transfer ratio could predict patients at risk for radiation necrosis after radiation therapy for medulloblastoma.
\end{abstract}

MATERIALS AND METHODS: Ninety-five eligible patients with medulloblastoma (41\% female; mean age, 11.0 [SD, 5.4] years) had baseline balanced steady-state free precession MR imaging before proton or photon radiation therapy. Associations among baseline supratentorial magnetization transfer ratio, radiation necrosis (spontaneously resolving/improving parenchymal enhancement within the radiation field) ${ }^{3}$, age, and the presence of visible brain metastases were explored by logistic regression and parametric/nonparametric techniques as appropriate.

RESULTS: Twenty-three of 95 (24.2\%) children (44\% female; mean age, 10.7 [SD, 6.7] years) developed radiation necrosis after radiation therapy (19 infratentorial, 1 supratentorial, 3 both). The mean pretreatment supratentorial WM magnetization transfer ratio was significantly lower in these children (43.18 versus $43.50, P=.03$ ). There was no association between the supratentorial WM magnetization transfer ratio and age, sex, risk/treatment stratum, or the presence of visible brain metastases.

CONCLUSIONS: A lower baseline supratentorial WM magnetization transfer ratio may indicate underlying structural WM susceptibility to radiation necrosis and may identify children at risk for developing radiation necrosis after craniospinal irradiation for medulloblastoma.

ABBREVIATIONS: bSSFP = balanced steady-state free precession; CTCAE = Common Terminology Criteria for Adverse Events; $M_{0}=$ non-MT-sensitized; $M_{M T}=M T$-sensitized; MT = magnetization transfer; MTR = magnetization transfer ratio; PFS = posterior fossa syndrome; RN = radiation necrosis; RT = radiation therapy; ST = supratentorial

adiation necrosis $(\mathrm{RN})$ is a relatively uncommon complication of radiation therapy in children with medulloblastoma,

Received August 2, 2021; accepted after revision October 20.

From the Department of Radiology (.H.H.), Dartmouth-Hitchcock Medical Center, Lebanon, New Hampshire; Geisel School of Medicine (J.H.H.), Dartmouth College, Hanover, New Hampshire; Departments of Diagnostic Imaging (P.Z., N.D.S., A.E.), Biostatistics (Y.H., Y.L.), Division of Neurology (R.B.K.), Department of Pediatrics, and Departments of Neuro-Oncology (A.G., G.R.) and Radiation Oncology (T.E.M.), St. Jude Children's Research Hospital, Memphis, Tennessee; Department of Radiology (O.B.) Division of Radiological Physics, University Hospital Basel, Basel, Switzerland; and Department of Biomedical Engineering (O.B), University of Basel, Allschwil, Switzerland. Drs Giles Robinson and Thomas Merchant are co-senior authors.

This work was supported, in part, by grant No. CA21765 from the National Cancer Institute and by the American Lebanese and Syrian Associated Charities.

Please address correspondence to Julie H. Harreld, MD, Department of Radiology, Dartmouth-Hitchcock Medical Center, Lebanon, NH 03766; e-mail:

Julie.H.Harreld@Hitchcock.org

- Indicates open access to non-subscribers at www.ajnr.org

Indicates article with online supplemental data.

http://dx.doi.org/10.3174/ajnr.A7393 with a reported incidence ranging from $19 \%$ to $>30 \% .{ }^{1-3}$ Current pathologic studies of $\mathrm{RN}$ suggest that damage to vascular endothelial cells and myelin-producing oligodendrocytes leads to increased vascular permeability, gliosis, and ultimately demyelination. ${ }^{4,5}$ Accordingly, abnormal posttreatment enhancement of nontumoral brain within the radiation field is the imaging hallmark of early radiation necrosis. ${ }^{1,2,6}$

For most patients with posterior fossa tumors, RN occurs in the posterior fossa within 1 year of radiation therapy (RT) (the "subacute" period), and symptoms are absent. ${ }^{1,2,4,7,8}$ Nevertheless, patients with medulloblastoma have shown significantly greater long-term decline in intelligence quotient and mathematic reasoning scores than identically treated patients without $\mathrm{RN}^{6}{ }^{6}$ The ability to predict which patients are likely to develop RN could be useful for implementing mitigation or heightened monitoring strategies. Unfortunately, there are no reliable clinical predictors of $\mathrm{RN}$ in this population, including age 


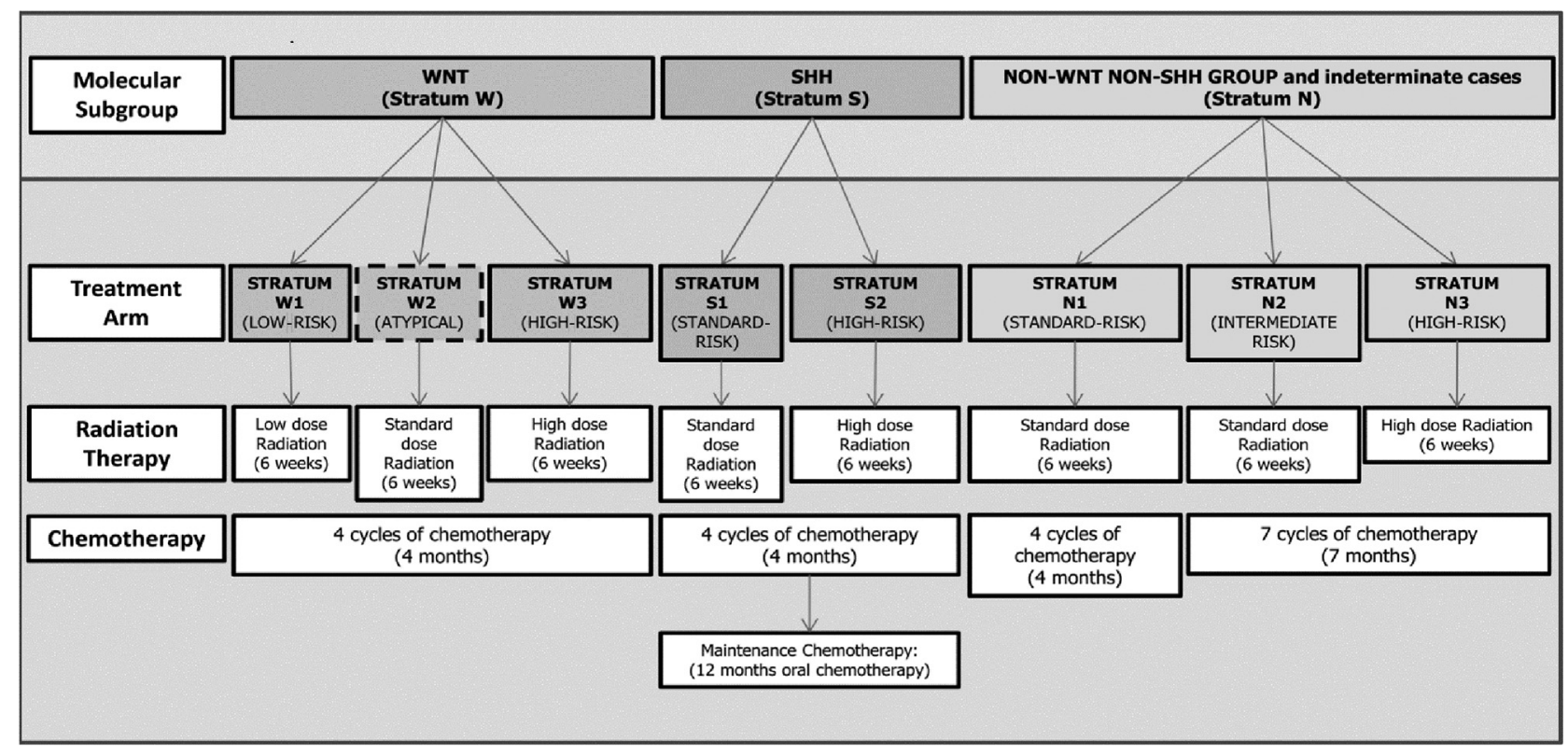

FIG 1. Risk-based treatment stratification schema. SHH indicates sonic hedgehog; WNT, wingless.

(in patients older than 3 years of age), ${ }^{1-3,7,9,10}$ chemotherapeutic regimen, ${ }^{1,10}$ metastatic status, ${ }^{1}$ or photon radiation dose/ volume. ${ }^{1,9}$

Magnetization transfer (MT) imaging quantifies the proportion of myelin ("myelin density") within a given volume of brain by calculating the ratio of images acquired with and without saturation of myelin/macromolecular protons, the magnetization transfer ratio (MTR). ${ }^{11-13}$ An advantage of the MTR over DTI for the study of WM in children is the insensitivity of the MTR to potentially confounding age-related structural changes detectable by DTI. ${ }^{13}$

We hypothesized that patients with lower WM myelin density before treatment would be more susceptible to radiation necrosis. We, therefore, investigated whether the pretreatment MTR of unperturbed supratentorial (ST) WM could predict development of radiation necrosis after $\mathrm{RT}$ in patients with medulloblastoma.

\section{MATERIALS AND METHODS}

This prospective study was performed with the approval of the St. Jude Children's Research Hospital IRB and informed parental or patient consent and/or assent. Between June 2013 and February 2019, one hundred sixty-four patients with previously untreated medulloblastoma were enrolled in a prospective clinical trial (www.clinicaltrials.gov, NCT01878617) at a single institution. Enrollment criteria included 3 years of age or older and younger than 22 years (younger than 40 years for sonic hedgehog medulloblastoma) at diagnosis.

All patients underwent attempted maximal tumor resection. Patients were stratified into low-, standard-, and high-risk treatment groups on the basis of the molecular subgroup, postsurgical tumor volume, histology, MYC/MYCN, and metastatic status (M0 to M4) (Fig 1). ${ }^{14,15}$ Treatment guidelines for risk-adapted photon or proton craniospinal and conformal primary (posterior fossa) site irradiation ranged from 15 to $51 \mathrm{~Gy}$ (craniospinal irradiation/primary site) for low-risk patients to 36 to $54 \mathrm{~Gy}$ for those at high risk, with additional boost of 45-50.4 Gy to metastatic sites when appropriate. Chemotherapy commenced 6 weeks post-RT (Fig 1).

One hundred twenty-five patients had baseline balanced steady-state free precession (bSSFP) MT imaging after surgery and before RT and chemotherapy. The subacute ("early delayed") period was defined as up to 1 year after completion of RT. . $^{1,4,8}$ Patients without $\mathrm{RN}$ and $<1$ year of follow-up imaging were, therefore, excluded. Patients who developed late effects ( $>1$ year after completing RT) were also excluded because late effects tend to be more clinically relevant and persistent and may differ in underlying pathologic mechanism. ${ }^{1,4,8}$ Follow-up diagnostic imaging was performed at 3-month intervals for the first 3 years, then at 6-month intervals for 3 years. Until March 16, 2015, MT imaging was performed in conjunction with fMRI before or within 2 weeks after beginning RT. After March 16, 2015, MT imaging was performed at the time of baseline, pretreatment diagnostic MR imaging.

3D bSSFP images were acquired on a 3T Magnetom Prisma or Magnetom Skyra scanner (Siemens) with a 64- or 20-channel head and neck coil. Parameters were $\alpha=30^{\circ}$, bandwidth $=789 \mathrm{~Hz} /$ pixel, FOV $=256 \times 256 \mathrm{~mm}$, matrix $=192 \times 192$, and section thickness $=$ $1.33 \mathrm{~mm}$, yielding $1.33-\mathrm{mm}$ isotropic voxels. TR/TE/radiofrequency pulse duration were 3.09/1.55/200 $\mathrm{ms}$ and 4.39/2.2/1500 ms for MTsensitized $\left(\mathrm{M}_{\mathrm{MT}}\right)$ and non-MT-sensitized $\left(\mathrm{M}_{0}\right)$ bSSFP sequences, respectively. Four examinations with legacy equipment Magnetom Tim Trio (Siemens) and head matrix coil (Siemens) were excluded from analysis.

Images were processed using in-house scripts in Matlab (MathWorks). MT images were coregistered with 3D T1weighted MPRAGE images $(\mathrm{TR} / \mathrm{TE} / \mathrm{TI}=1980 / 2.26 / 1000 \mathrm{~ms}$, $\mathrm{FOV}=256 \times 256 \mathrm{~mm}$, matrix $=256 / 256$, section thickness $=$ $1 \mathrm{~mm}$ ) for segmentation. ST WM and GM were segmented using related functions from SPM software (http://www.fil.ion.ucl.ac. 


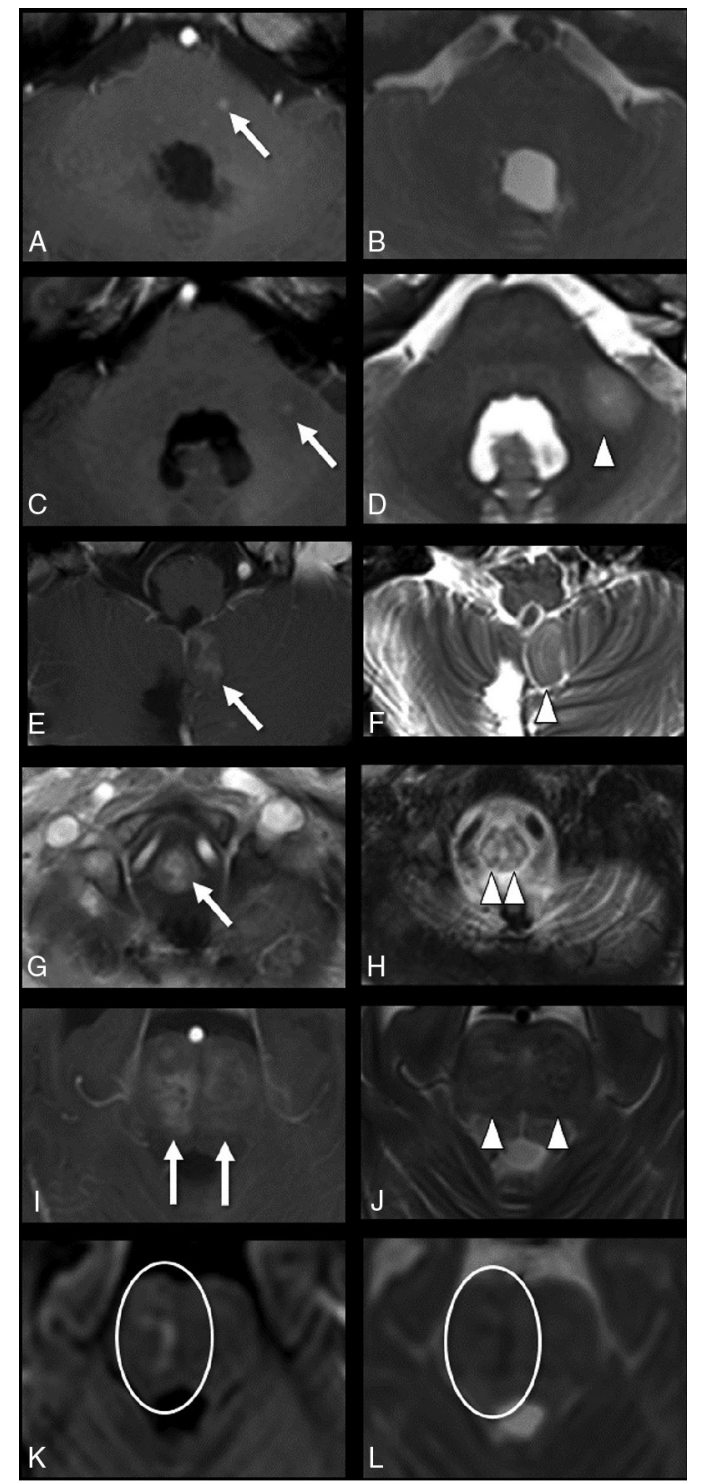

FIG 2. Image grading of radiation necrosis. Arrows show enhancement on postcontrast TIWI $(A, C, E, G, I)$, and arrowheads show edema on T2WI $(B, D, F, H, J)$. Grade 1: Punctate enhancement of the left pons $(A)$ with no edema on T2WI (B). Grade 2: Punctate enhancement of the left middle cerebellar peduncle $(C)$ with edema $(D)$. Grade 3: Confluent enhancement of the left cerebellar tonsil $(E)$ with ipsilateral edema $(F)$. Grade 4: Confluent enhancement involving the cervicomedullary junction bilaterally $(G)$, with bilateral edema $(H)$. Grade 5: Confluent enhancement (I) and edema (I) in the pons bilaterally ( () , with restricted diffusion evidenced by increased signal on DWI $(K)$ and low signal on the ADC map $(L)$.

$\mathrm{uk} / \mathrm{spm} / \mathrm{software} / \mathrm{spm} 12$ ) in Matlab (Mathworks, Inc.). MTR was calculated for ST WM in percentage units $(p u)$ as

$$
M T R=\frac{\left(M_{0}-M_{M T}\right)}{M_{0}} \times 100 p u .
$$

A board-certified neuroradiologist with a Certificate of Added Qualification and 10 years' experience interpreting pediatric oncologic neuroimaging prospectively reviewed all baseline MRIs for early delayed RN, defined as new brain parenchymal enhancement within the radiation field that improved or resolved without additional cancer-directed therapy. ${ }^{2,8}$ Because bulky leptomeningeal metastases could theoretically involve the WM and thereby alter the MTR, ST brain metastases were noted if present. Medical records for suspected cases of $\mathrm{RN}$ were reviewed by a pediatric oncologist (G.R.), and symptoms were graded according to the Common Terminology Criteria for Adverse Events (CTCAE), Version 4.03. ${ }^{16}$ All suspected cases of RN were reviewed for confirmation by a second board-certified neuroradiologist with a Certificate of Added Qualification and 15 years' experience in interpreting pediatric oncologic neuroimaging. Each confirmed case was then graded in consensus according to the following scale (Fig 2):

1) Punctate or stippled enhancement without edema

2) Punctate or stippled enhancement with edema (even if bilateral)

3) Nodular or confluent enhancement, some edema, does not cross the midline, $\mathrm{Or}$, nodular/confluent enhancement, no appreciable edema, crosses the midline

4) Confluent enhancement and edema, bilateral or crosses the midline for midline structures

5) Confluent enhancement and edema, restricted diffusion.

Times from completion of radiation therapy to the appearance of $\mathrm{RN}$ and from the appearance to resolution or improvement of $\mathrm{RN}$ were recorded.

\section{Statistical Analysis}

The associations between the development of RN and baseline ST WM MTR, patient age, risk group (low, standard, or high), tumor subtype, technique (proton versus photon), and the presence of visible brain metastases were tested using the Wilcoxon rank sum test (continuous variables) or the Fisher exact test (categoric variables). Associations between baseline ST WM MTR and age, sex, risk/treatment stratum, and the presence of visible brain metastases were further assessed with linear/logistic regression and adjusted for magnet and/or coil if appropriate. Maximum imaging and clinical grades were compared among modalities with the Fisher exact test. All statistical evaluations were performed with SAS, Version 9.4. (SAS Institute). A 2-sided significance level of $P<.05$ was considered statistically significant.

\section{RESULTS}

Of 125 eligible patients, 30 were excluded for technically inadequate imaging $(n=11)$, legacy equipment $(n=4)$, follow-up $<1$ year $(n=14)$, or RN $>1$ year after RT $(n=1)$. Of 95 patients included in the final analysis, 23 (24.2\%) developed RN (Online Supplemental Data). RN was initially noted on imaging a mean of 146.4 (SD, 58.2) days (range, 34-258 days) after treatment and resolved a mean of 152 (SD, 88.9) days (range, 49-364 days) after onset in 20 patients. Improvement without documented resolution was noted in 3 others: 2 who died of progressive disease (43 and 295 days' imaging follow-up) and another with persistent, though improved, imaging findings (937 days' follow-up).

Baseline MT imaging was acquired an average of 18.4 [SD, 10] days after surgery. ST WM MTR was significantly lower in children who later developed $\mathrm{RN}$ than those who did not $(P=.03$, Online Supplemental Data). Demographic and clinical characteristics, including risk/treatment stratum, did not differ (Online Supplemental Data). 
On logistic regression, there was no significant association of baseline ST WM MTR with the magnet $(P=.28)$. An association between baseline ST WM MTR and the coil approached significance $(P=.07)$. When we controlled for the coil, each unit decrease in ST WM MTR at baseline increased the odds of developing RN by $56 \%$ (estimate $=-0.8153, \mathrm{OR}=0.44, P=.04$ ). Also when we controlled for the coil, there was no association between ST MTR and age $(P=.41)$, sex $(P=.15)$, risk/treatment stratum $(P=.60)$, tumor subtype $(P=.67)$, or the presence of visible brain metastases $(P=.69)$.

$\mathrm{RN}$ was mild (grades 1-2) on imaging in 18/23 (78.3\%) patients and asymptomatic (CTCAE grade 1) in 20/23 (87.0\%). Three patients required intervention (CTCAE $\geq 2$ ). Patients with $\mathrm{RN}$ (12/23, 52.2\%) were more likely than those without RN (20/ $72,27.8 \%$ ) to have posterior fossa syndrome (PFS, $P=.02$ ), potentially confounding symptom assessment. There was no association between baseline ST WM MTR and PFS $(P=.93)$.

Most imaging changes were mild (grades 1 or 2, Fig 2). Cerebellar involvement was most frequent (12/23 patients, $52.2 \%$ ), and 22/23 (95.7\%) had infratentorial RN. Restricted diffusion (grade 5) was observed only in the spinal cord, cervicomedullary junction, pons, and middle cerebellar peduncle (Online Supplemental Data).

There was no significant difference in imaging grade, clinical grade, time to onset, or time to resolution of $\mathrm{RN}$ between patients who had photon or proton beam RT or focal (primary site) RT (Online Supplemental Data).

\section{DISCUSSION}

$\mathrm{RN}$ is an uncommon complication of radiation therapy in patients with medulloblastoma and is associated with long-term cognitive sequelae, despite occurring predominantly in the posterior fossa, remote from ST brain centers responsible for cognition. ${ }^{1}$

In this study, we found that decreased pretreatment MTR in normal ST WM predicted posttreatment RN in patients with newly diagnosed medulloblastoma undergoing RT. Baseline MTR was not associated with age, sex, medulloblastoma subtype, risk/treatment stratum, or metastatic status. As in a prior large medulloblastoma cohort, ${ }^{1}$ the incidence of subacute $\mathrm{RN}$ did not differ significantly by risk/treatment stratum or $\mathrm{M}$ stage. Our findings suggest that decreased myelin density, either intrinsic or secondary to tumor and/or surgery, may be a predisposing factor for $\mathrm{RN}$.

Although demyelination is a known consequence of radiation damage, ${ }^{4,5}$ there is currently no known association between myelin density and a predisposition to radiation injury. Our current understanding of genetic variations altering myelin development is driven by analysis in known syndromes. ${ }^{17}$ It is possible that other genetic differences in myelination, without clinical manifestations under normal circumstances, could predispose to radiation injury. The field of radiogenomics seeks to identify the genetic basis for differences in susceptibility to radiation injury among individuals. ${ }^{18}$ Our findings suggest that genes governing myelin formation could be a high-yield target for investigation.

A second possibility is that the lower pretreatment ST WM MTR we observed in patients with $\mathrm{RN}$ resulted from a greater disruption in the posterior fossa from tumor and surgery, sensitizing it to local radiation injury and manifesting as altered myelin density in the ST compartment via diaschisis.
Diaschisis describes a phenomenon in which a focal brain injury manifests as inflammation, degeneration, and demyelination in brain regions connected to, but remote from, the primary injury. ${ }^{19-21}$ Imaging studies showing decreased cortical perfusion ${ }^{22}$ and disruption of the cerebello-thalamo-cerebral WM tracts on $\mathrm{DTI}^{23}$ after cerebellar surgery support diaschisis as the mechanism underlying PFS, occurring in 39\%-40\% of children with medulloblastoma, with a mean latency of 1.7 days. ${ }^{24}$ Pathologic studies of brain injury in animals show inflammation and hydropic changes in remote diaschisis lesions. ${ }^{20,21}$ Such changes in the ST brain would be expected to reduce myelin density and, therefore, MTR.

The patients with $\mathrm{RN}$ in our series had a greater incidence of PFS (52.2\%) than those without RN (27.8\%), supporting an association between cerebellar injury and $\mathrm{RN}$. The lack of a direct relationship between baseline ST WM MTR and PFS, however, suggests that while surgery-related diaschisis lesions within affected WM tracts may contribute to radiation sensitivity, they are insufficient to explain the lower baseline ST WM MTR we observed in patients who later developed $\mathrm{RN}$.

This study was limited in scope, specifically targeting prediction of subacute (within 1 year post-RT), enhancing radiation injury $(\mathrm{RN})$ in patients with medulloblastoma who consented to and underwent baseline MT imaging. It may not, therefore, represent the overall incidence, frequency, or full spectrum of radiation injury in this population. Furthermore, we did not investigate the association between $\mathrm{RN}$ and patient-specific radiation dose or treatment volume, which could vary per the investigator's discretion. We had no reason to suspect a relationship between pretreatment MTR and the subsequent radiation dose; thus, this analysis was not relevant to this possibility. However, it is quite possible for RN to occur despite a normal MTR. While decreased MTR increases the odds of RN, it is not the sole predictor of this complex pathology. RN timing specificity was limited by imaging frequency, which was dictated by the clinical trial protocol except in rare symptomatic presentations requiring additional imaging. Finally, WM MTR values obtained with bSSFP are typically higher than those obtained with 3D MT-prepared spoiled gradient-recalled imaging, with which the values presented here should not be compared. ${ }^{25}$ bSSFP does, however, offer a markedly shorter imaging time and improved resolution and signal-to-noise ratio, important advantages over spoiled gradient-recalled imaging, particularly in children. ${ }^{25}$

\section{CONCLUSIONS}

Reduced MTR in normal-appearing ST WM at pretreatment baseline predicts the risk for radiation necrosis in patients with medulloblastoma, potentially facilitating heightened monitoring and mitigation strategies. Further validation and study of mechanisms underlying this finding are indicated.

\section{ACKNOWLEDGMENT}

The authors would like to acknowledge the invaluable contributions of Elizabeth Sanchez, BS, for keeping us on track even through a global pandemic with her meticulous organization, positive outlook, and unwavering dedication to our patients. 
Disclosure forms provided by the authors are available with the full text and PDF of this article at www.ajnr.org.

\section{REFERENCES}

1. Fouladi M, Chintagumpala M, Laningham FH, et al. White matter lesions detected by magnetic resonance imaging after radiotherapy and high-dose chemotherapy in children with medulloblastoma or primitive neuroectodermal tumor. J Clin Oncol 2004;22:4551-60 CrossRef Medline

2. Kralik SF, Ho CY, Finke W, et al. Radiation necrosis in pediatric patients with brain tumors treated with proton radiotherapy. AJNR Am J Neuroradiol 2015;36:1572-78 CrossRef Medline

3. Spreafico F, Gandola L, Marchiano A, et al. Brain magnetic resonance imaging after high-dose chemotherapy and radiotherapy for childhood brain tumors. Int J Radiat Oncol Biol Phys 2008;70:101119 CrossRef Medline

4. Belka C, Budach W, Kortmann RD, et al. Radiation induced CNS toxicity: molecular and cellular mechanisms. Br J Cancer 2001;85:123339 CrossRef Medline

5. Pruzincová L, Steno J, Srbecký M, et al. MR imaging of late radiation therapy- and chemotherapy-induced injury: a pictorial essay. Eur Radiol 2009;19:2716-27 CrossRef Medline

6. Muscal JA, Jones JY, Paulino AC, et al. Changes mimicking new leptomeningeal disease after intensity-modulated radiotherapy for medulloblastoma. Int J Radiat Oncol Biol Phys 2009;73:214-21 CrossRef Medline

7. Gunther JR, Sato M, Chintagumpala M, et al. Imaging changes in pediatric intracranial ependymoma patients treated with proton beam radiation therapy compared to intensity modulated radiation therapy. Int J Radiat Oncol Biol Phys 2015;93:54-63 CrossRef Medline

8. Ball WS Jr, Prenger EC, Ballard ET. Neurotoxicity of radio/chemotherapy in children: pathologic and MR correlation. AJNR Am J Neuroradiol 1992;13:761-76 Medline

9. Murphy ES, Merchant TE, Wu S, et al. Necrosis after craniospinal irradiation: results from a prospective series of children with central nervous system embryonal tumors. Int J Radiat Oncol Biol Phys 2012;83:e655-60 CrossRef Medline

10. Plimpton SR, Stence N, Hemenway M, et al. Cerebral radiation necrosis in pediatric patients. Pediatr Hematol Oncol 2015;32:78-83 CrossRef Medline

11. Bieri O, Scheffler K. Optimized balanced steady-state free precession magnetization transfer imaging. Magn Reson Med 2007;58:511-18 CrossRef Medline
12. Grossman RI, Gomori JM, Ramer KN, et al. Magnetization transfer: theory and clinical applications in neuroradiology. Radiographics 1994;14:279-90 CrossRef Medline

13. Moura LM, Kempton M, Barker G, et al. Age-effects in white matter using associated diffusion tensor imaging and magnetization transfer ratio during late childhood and early adolescence. Magn Reson Imaging 2016;34:529-34 CrossRef Medline

14. Chang $\mathrm{CH}$, Housepian EM, Herbert C Jr. An operative staging system and a megavoltage radiotherapeutic technic for cerebellar medulloblastomas. Radiology 1969;93:1351-59 CrossRef Medline

15. Gajjar AJ, Robinson GW. Medulloblastoma-translating discoveries from the bench to the bedside. Nat Rev Clin Oncol 2014;11:714-22 CrossRef Medline

16. Department of Health and Human Services National Institutes of Health, National Cancer Institute. Common Terminology Criteria for Adverse Events, Version 4.03. https://evs.nci.nih.gov/ftp1/CTCAE/ CTCAE_4.03/CTCAE_4.03_2010-06-14_QuickReference_5x7.pdf. Accessed December 17, 2020

17. Sarret C. Leukodystrophies and genetic leukoencephalopathies in children. Rev Neurol (Paris) 2020;176:10-19 CrossRef Medline

18. Brothwell MR, West CM, Dunning AM, et al. Radiogenomics in the era of advanced radiotherapy. Clin Oncol ( $R$ Coll Radiology) 2019;31:319-25 CrossRef Medline

19. Taylor DL, Joashi UC, Sarraf C, et al. Consequential apoptosis in the cerebellum following injury to the developing rat forebrain. Brain Pathol 2006;16:195-201 CrossRef Medline

20. Weishaupt N, Zhang A, Deziel RA, et al. Prefrontal ischemia in the rat leads to secondary damage and inflammation in remote gray and white matter regions. Front Neurosci 2016;10:81 CrossRef Medline

21. Wiley CA, Bissel SJ, Lesniak A, et al. Ultrastructure of diaschisis lesions after traumatic brain injury. J Neurotrauma 2016;33:186682 CrossRef Medline

22. Miller NG, Reddick WE, Kocak M, et al. Cerebellocerebral diaschisis is the likely mechanism of postsurgical posterior fossa syndrome in pediatric patients with midline cerebellar tumors. AJNR Am J Neuroradiol 2010;31:288-94 CrossRef Medline

23. Law N, Greenberg M, Bouffet E, et al. Clinical and neuroanatomical predictors of cerebellar mutism syndrome. Neuro Oncol 2012;14:1294-1303 CrossRef Medline

24. Toescu SM, Hales PW, Aquilina K, et al. Quantitative MRI in postoperative paediatric cerebellar mutism syndrome. Eur J Radiol 2018;108:43-51 CrossRef Medline

25. Garcia M, Gloor M, Bieri O, et al. MTR variations in normal adult brain structures using balanced steady-state free precession. Neuroradiology 2011;53:159-67 CrossRef Medline 